\title{
Adolescent salvia substance abuse
}

\author{
Sundeep Singh \\ University of Michigan Hospital, Department of Child and Adolescent Psychiatry, Ann Arbor, MI, USA
}

\section{ABSTRACT}

Background Salvia divinorum is a non-water-soluble hallucinogen that is becoming increasingly popular among adolescents. Salvia is a highly selective full agonist of primate and cloned human cerebral kappa-opioid receptors, although its psychotomimetic effects are similar to serotonergic agonists and NMDA glutamate antagonists. Salvia has been associated with depersonalization, laughter, feelings of levitation and self-consciousness. These effects resolve within 30 minutes following use. Salvia has been banned in many countries, although it remains legal and easily assessable over the internet in the United States. Case description A 15-year-old man with a history of salvia and marijuana use presented to psychiatric emergency services with acute onset of mental status changes characterized by paranoia, déjà vu, blunted affect, thought blocking and slow speech of 3 days' duration. Conclusion There is limited literature discussing the clinical effects of salvia use. Based on this case presentation, salvia use may be associated with many undocumented long-term effects such as déjà vu. The ease of use and increasing popularity of salvia requires further investigation into the clinical effects of salvia use.

Keywords Déjà vu, diviner's sage, salvia, Salvia divinorum, salvinorin A.

Correspondence to: Sundeep Singh, 3056 Vicksburg Dr, Lawrenceville, GA 30044, USA. E-mail: sundeeps@umich.edu Submitted 23 September 2006; initial review completed 6 November 2006; final version accepted 30 November 2006

Mr M, a 15-year-old man, presented to the psychiatric emergency services in May 2006 with an acute onset of mental status changes characterized by paranoia, déjà vu, blunted affect, thought blocking and slow speech of 3 days' duration. He had no previous medical history, but there was a family history of major depressive disorder in his mother and brother, who required in-patient hospitalization. The parents described a period beginning in early spring 2005, during which they became concerned about an episode characterized by anhedonia, depressed mood, declining academic performance and numerous absences from school. During May 2005, the mother considered seeking care from a psychologist for $\mathrm{Mr} \mathrm{M}$; however, his mood had improved significantly by late June without professional consultation. The temporal relationship of the subsequent events was difficult to ascertain, because the patient was a poor historian and resided on alternate weeks with each of his divorced parents. In July 2005, Mr $\mathrm{M}$ began using marijuana on a regular basis, along with a one-time use of mushrooms, presumed to contain psilocybin. In October, he placed an order online for Salvia divinorum, which he then smoked on multiple occasions over an unknown period of time. He described experienc- ing visual hallucinations involving vivid colors and objects when consuming salvia. When interviewed, neither he nor his family were able to recall the last time he engaged in the use of salvia. His family was able to confirm the use of marijuana several hours before his initial onset of paranoia 3 days prior to hospitalization. Before this admission, except for the depressive symptoms noted above, he had had no previous behavioral abnormalities noted by family and teachers.

During his hospitalization, he complained regularly of déjà vu without other concurrent visual or auditory hallucinations and paranoia towards those who informed him that he had not previously experienced events or conversations. His presentation was also notable for mental slowing, with poor short-term memory, attention and cognition. During his hospitalization all these symptoms improved significantly, although the feelings of déjà vu remained. The differential diagnosis for the cause of his déjà vu included salvia use, temporal lobe tumors or temporal lobe seizures. A magnetic resonance imaging (MRI) scan and electroecephalogram (EEG) obtained upon consulting with neurology found no notable abnormalities. The absence of any abnormalities in these 
studies and improving mental status during hospitalization were consistent with substance-induced mental status changes. Because marijuana has not been documented to be a cause of déjà vu, salvia was considered the most probable cause of déjà vu in Mr M.

$S$. divinorum, also known as magic mint or Diviner's Sage, was used by the shamans of Mazatec Indians in Oaxaca, Mexico for ritualistic purposes [1]. Recently, it is becoming an increasingly popular hallucinogen among adolescents. At least six different compounds (salvinorin A-F) have been extracted from the plant leaves with salvinorin A (Divinorin A) being the most psychoactive compound [2]. Salvinorin A is a non-water-soluble compound that is absorbed primarily by the respiratory tract and to a lesser extent by the oral mucosa when users chew or smoke dried leaves [3]. Salvinorin A is a highly selective full agonist of primate and cloned human cerebral kappa-opioid receptors, although its psychotomimetic effects are similar to serotonergic agonists (LSD) and NMDA glutamate antagonists (PCP) [4,5]. This association between psychotomimetic effects and kappaagonists has been documented with other substances [6]. Users typically describe experiencing depersonalization, hysterical laughter, transforming into people or animals, being in multiple places at the same time, levitation and increased sense of self-consciousness [7]. Users also indicate that these effects start within 5 minutes of consuming salvia and subside within 30 minutes [8]. Currently, there are no records of longterm side effects of salvia use.

Although this substance is available easily over the internet, it has been outlawed in Australia, Finland, Denmark, Spain and Norway. Similar to our patient, users are typically adolescent experimenters seeking a safe method for meditative introspection. Analogous to Bucheler et al.'s case report [1], our patient indicated that he felt he was well informed, and he thought salvia was a safe alternative to marijuana after reading websites citing many scientific articles. Although Mr M perceived that salvia's drug safety was well studied, only three studies and two case reports involving a total of 56 people discuss the effects of salvia in human subjects. Bucheler notes that the user may experience amnesia of the event, thus potentially contributing to our patient's inability to recollect his last use. As a non-water-soluble hallucinogen, salvia could potentially have long-term consequences associated with re-experiencing hallucinations or other neuropsychiatric sequelae such as déjà vu. By being accessible easily over the internet and becoming increasingly popular among adolescents around the world, salvia introduces a new substance about which healthcare workers should be informed, and the long-term effects should be evaluated.

Informed consent by the patient was obtained prior to publication of this case report.

\section{References}

1. Bucheler R., Gleiter C. H., Schwoerer P., Gaertner I. Use of non-prohibited hallucinogenic plants: increasing relevance for public health? Pharmacopsychiatry 2005; 38: 1-5.

2. Ortega A., Blount J. F., Machand P. S. Salvinorin: a new trans-neoclerodane diterpine from Salvia divinorum. J Chem Soc Perkin Trans 1982; 1: 2503-8.

3. Valdes L. J. III. Salvia divinorum and the unique diterpine hallucinogen salvinorin. J Psychoact Drugs 1994; 26: 277-83.

4. Roth B. L., Baner K., Westkaemper R., Siebert D., Rice K. C., Steinberg S. et al. Salvinorin A: a potent naturally occurring nonnitrogenous opioid selective agonist. Proc Natl Acad Sci USA 2002; 99: 11934-9.

5. Butelman E. R., Harris T. J., Kreek M. J. The plant derived hallucinogen, salvinorin A, produces $\kappa$-opioid agonist-like discriminative effects in rhesus monkeys. Psychopharmacology 2004; 172: 220-4.

6. Pfeiffer A., Brantl V., Herz L., Emrich H. M. Psychotomimesis mediated by к opiate receptors. Science 1986; 233: 774-6.

7. Gonzalez D., Riba J., Bouso J. C., Gomez-Jarabo G., Barbanoj M. J. Pattern of use and subjective effects of Salvia divinorum among recreational users. Drug Alcohol Depend 2006; 85: 157-62.

8. Siebert D. Salvia divinorum and salvinorum A: new pharmacologic findings. J Ethnopharmacol 1994; 43: 53-6. 